\title{
Mothers dealt with incompatible expectations during breast feeding and weaning
}

Hauck YL, Irurita VF. Constructing compatibility: managing breast-feeding and weaning from the mother's perspective. Qual Health Res 2002;12:897-914.

\section{QUESTION: What are women's experiences and perceptions during the process of managing the later stages of established breast feeding and ultimately weaning the child from the breast?}

Design

Grounded theory.

\section{Setting}

Social and cultural context of Western Australia.

\section{Participants}

33 mothers (mean age 33 y) mainly recruited from a larger study on breast feeding and weaning, who had each weaned a child within the previous 6 months. 17 of the women were first time mothers, and the remaining 16 had $\geq 2$ children.

\section{Methods}

Data were obtained from indepth interviews with 33 women, field notes, 9 postal questionnaires from fathers, interviews with 5 child health nurses, and a group interview with 7 child health nurses.

\section{Main findings}

Participants viewed their performance of breast feeding and weaning as a component of their whole mothering experience and ultimately wanted to be able to see themselves, and be seen by others, as good mothers.

2 major categories emerged from the data. The first category, incompatible expectations, occurred when mothers noted differing and often conflicting expectations between themselves and others such as partners or health professionals. Participants were caught in the middle of the contradictory and confusing debate about the best way to manage breast feeding and weaning.

The second major category, constructing compatibility by adapting focus, evolved in response to the incompatible expectations and involved 3 phases: shifting focus, selective focusing, and confirming focus. Shifting focus. Each participant reached a turning point when she decided something had to be done because she was no longer prepared to endure the confusion, self doubt, and guilt she felt because of incompatible expectations. Participants then clarified and/or modified the relative importance assigned to aspects of their breastfeeding and weaning. They then could take charge of their breast feeding experience by reinforcing personal expectations and goals. Selective focusing. Selective focusing involved mothers choosing to focus on others' expectations of breast feeding and weaning that matched their own beliefs. 2 strategies, embracing and distancing, were used. Embracing involved seeking and welcoming agreement from sources whose expectations were compatible with mothers' expectations, such as partner's reinforcement of weaning decisions. Most mothers also chose to distance themselves from disagreement with their decisions by either avoiding such situations or accepting the fact that incompatibilities would occur and dismissing or ignoring the influence of that source, even if the source was a health professional. Confirming focus. Once mothers had weaned their child from the breast, they focused on the positive outcomes of their decisions, such as having a more settled baby. Each woman acknowledged the emotional meaning of breast feeding, and "letting go" was an important aspect of adapting to a non-breastfeeding state.

\section{Conclusion}

Mothers revealed a common social problem of incompatible expectations, faced a dilemma in the management of their experience when personal expectations were in opposition to others' expectations, and used strategies to compensate for this incompatibility.

\section{COMMENTARY}

Breastfeeding promotion has focused on encouraging mothers to breast feed for a prescribed duration of time, usually according to government targets rather than the mother's own wishes. The study by Hauck and Irurita shows that it may be better to support mothers in their decisions, while attempts are made to change societal attitudes and expectations so that breast feeding is accepted as a normal behaviour. Breast feeding was inextricably linked with good mothering and perceptions of being a good mother. Mothers who did not conform to society's expectations experienced confusion, self doubt, guilt, or anger, which likely influenced their own beliefs about themselves as mothers. It is therefore important that mothers have a positive perception of their own breastfeeding experience to empower them to deal with the later challenges of mothering.

Clinical guidelines suggest that breast feeding should continue for $\geq 6$ months and thereafter as long as the mother and infant wish. ${ }^{1}$ The fact that all participants in this study had incompatible expectations shows the continuing effect of society's expectations on breastfeeding women. The mothers took steps to dismiss or avoid conflicting or opposing situations, and health professionals should consider realigning themselves to a "woman centred" rather than "breastfeeding centred" role. A woman centred advocate who supports a mother's own decisions and helps her to feel positive about herself and her role as a mother would be more effective than a breastfeeding centred advocate.

Participants were part of a larger study, but no information was given about the aims of this study, which might have influenced the responses given during interviews. Infants had been breast fed for 6 weeks to 6 years. It was sometimes unclear whether mothers were referring to a social expectation to continue breast feeding (of younger infants) or a social expectation to stop breast feeding (of older children). This study was done in a country where breast feeding was expected, and as the authors acknowledge, some of the information generated may not be relevant to other social and cultural contexts. It is likely, however, that the general themes are important for planning breastfeeding support programmes. Rhona McInnes, RGN, RM, PhD Lecturer, School of Nursing and Midwifery University of Glasgow, Glasgow, UK of Nursing and Public Health, Edith Cowan University, Perth,

Western Australia. y.hauck@ecu.edu.au

American Academy of Pediatrics. Work Group on Breastfeeding. Breastfeeding and the use of human milk. Pediatrics 1997;100:1035-9. 